\title{
CROSS-LAYER CONGESTION CONTROL, SCHEDULING AND POWER CONTROL DESIGN IN MULTIHOP NETWORKS WITH RANDOM ACCESS
}

\author{
Chengnian Long ${ }^{1,2}$, Xinping Guan ${ }^{2}, B o \mathrm{Li}^{l}$ \\ ${ }^{1}$ Department of Computer Science \\ Hong Kong University of Science and Technology \\ Clear Water Bay, Kowloon, Hong Kong, China \\ ${ }^{2}$ Department of Electrical Engineering \\ Yanshan University \\ Qinhuangdao, 066004, Hebei Province, P.R. China
}

\begin{abstract}
This paper considers optimal cross-layer rate control, scheduling design and power control for multi-hop wireless networks. The problem of optimal rate control, link scheduling, and link transmission power for all active time slot is formulated as a network utility maximization problem. In wireless multi-hop networks, the link capacity is a function of link scheduling and transmission power with time-varying and nonlinear properties. Those characteristic poses much challenge in joint design. To solve the non-convex and non-separable nonlinear program problem, a two time-scale distributed optimization approach is presented. By dual decomposition and gradient method, the NUM problem naturally decomposes into three subproblems: congestion control, scheduling design and power control. They interact through congestion price. The global convergence of this algorithm is proven. This paper presents a step towards a systematic approach to jointly design TCP congestion control algorithms, scheduling design and power control.
\end{abstract}

\section{INTRODUCTION}

Cross-layer design is becoming increasingly important for improving the performance of multihop wireless networks (see, e.g. [1], [2], [3] and cited reference therein). By simultaneously optimizing the control across multiple layers of the network, cross-layer design can substantially increase the end-to-end throughput and link capacity, reduce interference and power consumption. In this paper, we focus on the interaction of rate control at the transport layer, link scheduling and power control. Our goal is to present a systematic approach to jointly design TCP congestion control algorithms, random link access probabilities and transmission powers, not only to improve performance, but more importantly, to make their interaction more transparent.

The need for joint design across these three layers is motivated by the following three observations. First, a wireless network uses a shared medium, with interference across the logic links. If contentions among transmissions on different links are not appropriately controlled, a large number of collisions may occur, resulting in waste of resources such as bandwidth and energy, as well as loss of system efficiency and degradation of resource sharing fairness. Thus, in ad hoc wireless networks, the contention relations between link-layer flows provide fundamental constraints for resource allocation. Second, the attainable link active rates in a time slot on wireless links depend on the interference levels, which in turn depend on the power control policy [4]. Therefore, the link capacity is a combinatorial function for link scheduling and transmission power with time-varying and nonlinear properties. Third, TCP congestion control algorithms can be interpreted as distributed primal-dual algorithms over the Internet to maximize the aggregate utility. This series of work implicitly assumes a wired network where link capacities are fixed and shared by flows that traverse common links. A natural formulation for the joint design of congestion control, link scheduling and power control is then the utility maximization framework with new constraints that arise from channel contention and physical interference. In this paper, we extend the work in [5] to joint design across end-to-end rate control, random access protocol and power control. Because the link capacities constraints are non-convex and non-separable, the optimal solution is separated by two steps. The first step is to optimal rate control design with a fixed link capacity. The subproblem is reduced to classical TCP congestion control. The second step is based on gradient method to update the link access and power control protocol. 


\section{SYSTEM MODELING AND NOTATION}

A multihop wireless network can be represented as a directed graph $G=(V, L)$. Here $V$ represents the set of nodes in the network and $L$ the set of directed edges (links) in the network. The nodes communicate with each other via wireless links. Each node can communicate directly with a subset of other nodes. Assume the network is shared by a set $S$ of source indexed by $s$. A sequence of connected links $l \in L(s)$ forms a route originating from source $s$, and $f_{l}$ be the link attainable capacity. Since wireless links use a shared medium, they may interfere with each other if they transmit data at the same time. If the interference level between two links is too high, transmissions from those two links will collide, resulting in a failure of the transmission. In the following, we present the detailed contention relation based on the link layer model. In general, the link capacity $f_{l}$ can be represented as the function of power consumption and link access probability in a random access network. If the link interference can be restrained in link scheduling, the active rate $c_{l}$ in a time slot with an Additive White Gaussian Noise (AWGN) channel is

$$
c_{l}=W \log _{2}\left(1+P_{l} / N_{0} W\right) \text {, }
$$

where $W$ is the channel bandwidth, $N_{0}$ the noise spectrum density and $P_{l}$ transmission power. In the following, we present the random access protocol with neighborhood link conflict information. We define $L_{\text {out }}(v)$ as a set of outgoing links from node $v, L_{i n}(v)$ as a set of incoming links to node $v, t_{l}$ as the transmitter node of link $l$, and $r_{l}$ as the receiver node of link $l$. We also define $N_{t o}^{I}(l)$ as the set of nodes whose transmission cause interference to the receiver of link $l$, excluding the transmitter node of link $l$ and $L_{\text {from }}^{I}(v)$ as the set of links whose transmission get interfered from the transmission of node $v$, excluding outgoing links form node $v$. These two sets, $N_{t o}^{I}(l)$ and $L_{\text {from }}^{I}(v)$, can be obtained through the bipartite graph. Each node $v$ transmits data with a probability $\mathfrak{I}_{v}$. When it determines to transmit data, it choose one of its outgoing links with probability $q_{l} / \mathfrak{I}_{v}, \forall l \in L_{\text {out }}(v)$ such that $\sum_{l \in L_{\text {out }}(v)} q_{l} / \mathfrak{I}_{v}=1$ and transmits data only on the chosen link. Hence there is no collision among links that have the same transmitter node. Link $l, l \in L_{\text {out }}(v)$, transmits data with the persistence probability $q_{l}$ such that $\sum_{l \in L_{\text {out }}(v)} q_{l}=\mathfrak{I}_{v}, \forall v$. The link attainable capacity is

$$
f_{l}(\mathbf{q}, \mathbf{P})=c_{l} q_{l} \prod_{k \in N_{t o}^{l}(l)}\left(1-\mathfrak{I}_{k}\right) .
$$

\section{TWO-TIME SCALE DISTRIBUTED OPTIMIZATION APPROACH (TTSDOA)}

The network utility maximization (NUM) problem with "elastic" link capacities can be formulated as

$$
\begin{aligned}
\operatorname{maximize} & \sum_{s} \omega_{s} \log \left(x_{s}\right) \\
\text { subject to } & \sum_{s: l \in L(s)} x_{s} \leq f_{l}(q, P) \\
\mathrm{P} 1: & f_{l}(q \cdot P)=c_{l} q_{l} \prod_{k \in N_{t o}^{l}(l)}\left(1-\mathfrak{I}_{k}\right), \forall l \\
& \sum_{l \in L_{\text {out }}(v)} q_{l}=\mathfrak{I}_{v}, \forall v \\
& \mathbf{0} \_\mathbf{q} \_\mathbf{1}, \mathbf{0} \leq \mathfrak{I} \preceq \mathbf{1}, \mathbf{x}, \mathbf{P} \succeq \mathbf{0}
\end{aligned}
$$

The first and second sets of constraints ensure that the total source rates of traffic in a link cannot exceed the attainable rate of the link. The objective of this problem is to obtain the optimal persistence probabilities $\mathbf{q}$, transmit powers $\mathbf{P}$ on links and access probabilities $\mathfrak{I}$ for nodes that maximize the network utility. The rate control question therefore represents a joint optimization problem, which couples the link persistent probabilities at link layer, transmit powers at the physical layer with the source rates at the transport layer. Note that the NUM problem (3) is non-convex and non-separable. The major challenges are the global dependent between the source rates $\mathbf{x}$ and link capacities $\mathbf{f}$ and the nonlinear couple among link capacities $\mathbf{f}$, link persistent probabilities $\mathbf{q}$ and link transmit powers $\mathbf{P}$. Our primary goal in this paper is to distributively find the joint and globally optimal solution $\left(\mathbf{x}^{*}, \mathbf{q}^{*}, \mathbf{P}^{*}\right)$ to problem P1 by breaking down the global dependent and nonlinear couple limitation. In the following, we will present a two-time scale distributed optimization approach based on dual theory. We now outline the overall strategy to obtain the optimum solution for nonlinear program problem P1. Given fixed $f$, the problem P1 can be reduced to the following problem $\mathrm{P} 2$

$$
\hat{\psi}(f)=\max \left\{\sum_{s} \omega_{s} \log \left(x_{s}\right) \mid \sum_{s: l \in L(s)} x_{s} \leq f_{l}, \forall l, \mathbf{x} \succeq 0\right\}
$$

The optimum solution for problem P2 is parameterized by link capacities $\mathbf{f}$. We also can define function $\hat{\psi}(\mathbf{q}, \mathbf{P})=\hat{\psi}(f(\mathbf{q}, \mathbf{P}))$ for the link capacities. Let

$$
\psi(\mathbf{q}, \mathbf{P})=\max \left\{\sum_{s} \omega_{s} \log \left(x_{s}\right) \mid \text { constraint conditions in (3) }\right\},
$$

Hence $\psi(\mathbf{q}, \mathbf{P})=\max \{\tilde{\psi}(\mathbf{q}, \mathbf{P}) \mid \mathbf{0} \measuredangle \mathbf{q} \preceq \mathbf{1}, \mathbf{P} \succeq \mathbf{0}\}$. Then the local optimum solution for problem P1 can be obtained by the following steps:

(1). For problem P2, we introduce the Lagrangian

$$
L(\mathbf{x}, \boldsymbol{\lambda})=\sum_{s}\left(\omega_{s} \log \left(x_{s}\right)-\sum_{l \in L(s)} \lambda_{l} x_{s}\right)+\sum_{l} \lambda_{l} f_{l}(q, P)
$$


Consider the dual problem to the primal problem $\mathrm{P} 2$

$$
D: \min _{\lambda \succeq \underline{0}} D(\lambda)
$$

where

$$
\begin{aligned}
D(\boldsymbol{\lambda}) & =\max L(\mathbf{x}, \boldsymbol{\lambda}) \\
& =\sum_{s} \max _{x_{s} \geq 0}\left(\omega_{S} \log \left(x_{s}\right)-\sum_{l \in L(s)} \lambda_{l} x_{s}\right)+\sum_{l} \lambda_{l} f_{l}(q, P)
\end{aligned}
$$

The above congestion control problem is already simplicity solved by the TCP congestion problem, such as the TCP Vegas. With the TCP Vegas, the congestion control problem (6) admits a unique maximizer in time slot $t$

$$
\bar{x}_{s}(\bar{\lambda})=\arg \max _{x_{s} \geq 0} \sum_{s}\left(\omega_{s} \log x_{s}-\sum_{l \in L(s)} \lambda_{l} x_{s}\right)=\frac{\omega_{\mathrm{s}}}{\sum_{l \in L(s)} \bar{\lambda}_{l}}
$$

(2) In each time slot $t$, each link computes the gradient direction $\nabla_{\mathbf{q}} \bar{\psi}(\mathbf{q}, \mathbf{P})$ and $\nabla_{\mathbf{P}} \bar{\psi}(\mathbf{q}, \mathbf{P})$ at $\bar{x}_{s}(\bar{\lambda})$ as follows

$$
\begin{aligned}
& \nabla_{\mathbf{q}} \bar{\psi}(\mathbf{q}, \mathbf{P})=\sum_{j \in L} \bar{\lambda}_{j} \frac{\partial f_{j}}{\partial q_{l}}(\mathbf{q}(t), \mathbf{P}(t)) \\
& \nabla_{\mathbf{P}} \bar{\psi}(\mathbf{q}, \mathbf{P})=\sum_{j \in L} \bar{\lambda}_{j} \frac{\partial f_{j}}{\partial P_{l}}(\mathbf{q}(t), \mathbf{P}(t))
\end{aligned}
$$

where

$$
\begin{gathered}
\frac{\partial f_{j}(\mathbf{q}(t), \mathbf{P}(t))}{\partial q_{l}}=\left\{\begin{array}{lr}
c_{l} \prod_{k \in N_{t o}^{I}(l)}\left(1-\mathfrak{I}_{k}\right) & \text { if } j=l \\
-c_{j} q_{j} \prod_{k \in N_{t o}^{I}(j) \backslash\left\{t_{l}\right\}}\left(1-\mathfrak{I}_{k}\right) & \text { if } j \in L_{\text {from }}^{I}\left(t_{l}\right) \\
0 & \text { otherwise }
\end{array}\right. \\
\frac{\partial f_{l}}{\partial P_{l}}(\mathbf{q}(t), \mathbf{P}(t))=\frac{q_{l} \prod_{k \in N_{t o}^{I}(l)}\left(1-\mathfrak{I}_{k}\right)}{\log 2\left(N_{0}+P_{l} / W\right)} .
\end{gathered}
$$

Therefore, the link persistent probabilities and transmission powers can be updated in the gradient direction

$$
\begin{gathered}
q_{l}(t+1)=q_{l}(t)+\mu \sum_{j \in L} \bar{\lambda}_{j} \frac{\partial f_{j}}{\partial q_{l}}(\mathbf{q}(t), \mathbf{P}(t)), \\
P_{l}(t+1)=P_{l}(t)+\mu \bar{\lambda}_{l} \frac{\partial f_{l}}{\partial P_{l}}(\mathbf{q}(t), \mathbf{P}(t)) .
\end{gathered}
$$

Note that each link and its transmitter node only need to know the set of nodes whose transmission cause interference to the receiver of link and links that are interfered by its transmitter node in the link persistent probabilities update algorithm (9) and transmission powers update algorithm (10). There is no need for any additional information or message passing. Hence, it is local and distributed.

\section{CONVERGENCE ANALYSIS}

In this section, we prove the convergence property of the two time-scales distributed optimization Algorithm. Intuitively, the slow system (8)-(10) updates the link persistent probabilities and transmission power in the gradient direction. Therefore we can state the following local convergence property.

Theorem 1 The iterative algorithm with TTSDOA converges to the local optimum point of problem Pl when the step size $\alpha, \gamma$ is small enough.

We further show that, although nonlinear program problem P1 appears to be non-convex, its KKT points are globally optimum pomts by a bridge between the KKT points of problem $\mathrm{P} 1$ and that of a converted convex program with $\log$ transformation. First, the non-convex optimization problem can be easily converted into a convex optimization problem after the log transformation. The following Lemma states that the weighted proportionally fair rate control can be obtained by solving a convex optimization problem.

Lemma 1 The end-to-end proportionally fair rate control problem in a multihop random access network, as given by (3), is equivalent to the following convex programming problem $Q$ :

$$
\begin{array}{ll}
\operatorname{maximize} & \sum_{s} y_{s} \\
\text { subject to } & \log \left(\sum_{s: l \in l(s)} e^{y_{s} / \omega_{s}}\right)-\log \left(W \log _{2}\left(1+P_{l} / N_{0} W\right)\right) \\
& -\log q_{l}-\log \sum_{k \in N_{t o}^{l}(l)}\left(1-\mathfrak{I}_{k}\right) \leq 0, \forall l \\
& \sum_{l \in L_{\text {out }}(v)} q_{l}=\mathfrak{I}_{v}, \quad \forall v \\
& \mathbf{0} \_\mathbf{q} \preceq \mathbf{1}, \mathbf{0} \leq \mathfrak{I} \leq \mathbf{1}, \mathbf{x}, \mathbf{P} \succeq \mathbf{0}
\end{array}
$$

where $y_{s}$ is the weighted logarithm of the source rate $x_{s}$.

It is worth noting that we should not solve the either problem Q or P1 directly. Using the similar idea in [5], we present the optimization solution property between the problem P1 and problem Q by the following Lemma.

Lemma 2 If $x_{s}{ }^{*}, s=1, \cdots,|S|, q_{l}^{*}$, and $P_{l}^{*}, l \in L$ satisfy the first order necessary condition for optimality for nonlinear program in (3), then $y_{s}^{*}=\log \left(x_{s}^{*}\right), s=1, \cdots,|S|, q_{l}^{*}$, and $P_{l}^{*}, l \in L$ will be the global optimum points for the convex program in (11). Conversely, if $y_{s}^{*}, s=1, \cdots,|S|, q_{l}^{*}$, and $P_{l}^{*}, l \in L$ satisfy the first order necessary condition for optimality for the convex program in (11), then $x_{s}^{*}=e^{y_{s}}, s=1, \cdots,|S|, q_{l}^{*}$, and $P_{l}^{*}, l \in L$ will satisfy the first order necessary condition for optimality for nonlinear program in (3).

Proof: Denote $\mathbf{x}^{*}=\left(x_{s}, s \in S\right), \mathbf{y}^{*}=\left(y_{s}^{*}=\log \left(x_{s}^{*}\right), s \in S\right)$,

$$
\begin{aligned}
\mathbf{q}^{*} & =\left(q_{l}^{*}, l \in L\right), \mathbf{P}^{*}=\left(P_{l}^{*}, l \in L\right), \\
g_{l} & =\sum_{s: l \in L(s)} x_{s}-f_{l} \\
& =\sum_{s: l \in L(s)} x_{s}-W \log _{2}\left(1+p_{l} / N_{0} W\right) q_{l} \prod_{k \in N_{t 0}^{l}(l)}\left(1-\mathfrak{I}_{k}\right)
\end{aligned}
$$




$$
\begin{aligned}
\tilde{g}_{l}= & \log \left(\sum_{s: l \in L(s)} e^{y_{s}}\right)-\log \left(f_{l}\right)=\log \left(\sum_{s: l \in L(s)} e^{y_{s}}\right)- \\
& \log \left(W \log _{2}\left(1+p_{l} / N_{0} W\right)-\log q_{l}-\log \sum_{k \in N_{t_{0}}^{I}(t)}\left(1-\mathfrak{I}_{k}\right)\right. \\
\pi= & \left\{l:\left.g_{l}\right|_{x^{*}, q^{*}, p^{*}}=0, l \in L\right\}, \tilde{\pi}=\left\{l:\left.\tilde{g}_{l}\right|_{x^{*}, q^{*}, p^{*}}=0, l \in L\right\},
\end{aligned}
$$

If $\mathbf{x}^{*}, \mathbf{q}^{*}, \mathbf{P}^{*}$ satisfy the first order necessary condition, then

there exists $\vartheta_{l}^{*} \geq 0 \quad$ for $\quad l \in \pi \quad$ such that $\left.\left(-\nabla U+\sum_{l \in \pi} \tilde{\vartheta}_{l}^{*} \nabla g_{l}\right)\right|_{\mathbf{x}^{*}, \mathbf{q}^{*}, \mathbf{P}^{*}}=0$. Therefore, for $x_{s}$, we have

$$
\left.\left(-\frac{\partial U}{\partial x_{s}}+\sum_{l \in \pi} \tilde{\vartheta}_{l}^{*} \frac{\partial g_{l}}{\partial x_{s}}\right)\right|_{x^{*}, q^{*}, p^{*}}=\left(-\frac{1}{x_{s}}+\left.\sum_{l \in \pi \cap L(s)} \tilde{\vartheta}_{l}^{*}\right|_{x^{*}, q^{*}, p^{*}}=0\right.
$$

For $q_{l}$, we have

$$
\left.\left(-\frac{\partial U}{\partial q_{l}}+\sum_{l \in \pi} \tilde{\vartheta}_{l}^{*} \frac{\partial g_{l}}{\partial q_{l}}\right)\right|_{x^{*}, q^{*}, p^{*}}=\left.\left(\sum_{l \in \pi} \tilde{\vartheta}_{l}^{*} \frac{\partial f_{l}}{\partial q_{l}}\right)\right|_{x^{*}, q^{*}, p^{*}}=0
$$

and for $P_{l}$ we have

$$
\left.\left(-\frac{\partial U}{\partial P_{l}}+\sum_{l \in \pi} \tilde{\vartheta}_{l}^{*} \frac{\partial g_{l}}{\partial P_{l}}\right)\right|_{x^{*}, q^{*}, p^{*}}=\left.\left(\sum_{l \in \pi} \tilde{\vartheta}_{l}^{*} \frac{\partial f_{l}}{\partial P_{l}}\right)\right|_{x^{*}, q^{*}, p^{*}}=0
$$

For the nonlinear program in (11), we take $\tilde{\vartheta}_{l}^{*}=\vartheta_{l}^{*} \cdot f_{l}\left(q^{*}, P^{*}\right)$ for all $l$ in $\tilde{\pi}$. Note that $\pi=\tilde{\pi}$ and hence $\left.g_{l}\right|_{x^{*}, q^{*}, p^{*}}=0$ for any $l$ in $\tilde{\pi}$. It then follows $f_{l}\left(q^{*}, P^{*}\right)=\sum_{s: l \in L(s)} e^{y_{s^{*}}}$. For $y_{s}$, we have

$$
\begin{aligned}
& \left.\left(-\frac{\partial U}{\partial y_{s}}+\sum_{l \in \bar{\pi}} \tilde{\vartheta}_{l}^{*} \frac{\partial \tilde{g}_{l}}{\partial y_{s}}\right)\right|_{x^{*}, q^{*}, p^{*}}=\left.\left(\sum_{l \in \bar{\pi} \cap L(s)}^{*} \frac{\tilde{\vartheta}_{l}^{*} e^{y s}}{\sum_{s: l \in L(s)} e^{y s}}-1\right)\right|_{x^{*}, q^{*}, p^{*}} \\
& =\left.\left(-1+\sum_{l \in \bar{\pi} \cap L(s)}^{*} \tilde{\vartheta}_{l}^{*} x_{s}\right)\right|_{x^{*}, q^{*}, p^{*}}=\left.x_{s}\left(-\frac{1}{x_{s}}+\sum_{l \in \pi \cap L(s)}^{*} \tilde{\vartheta}_{l}^{*}\right)\right|_{x^{*}, q^{*}, p^{*}}=0
\end{aligned}
$$

For $q_{l}$, we have

$$
\begin{aligned}
& \left.\left(-\frac{\partial U}{\partial q_{l}}+\sum_{l \in \tilde{\pi}} \tilde{\vartheta}_{l}^{*} \frac{\partial \tilde{g}_{l}}{\partial q_{l}}\right)\right|_{x^{*}, q^{*}, p^{*}}=\left.\left(\sum_{l \in \tilde{\pi}} \tilde{\vartheta}_{l}^{*} \frac{1}{f_{l}} \frac{\partial f_{l}}{\partial s_{l}}\right)\right|_{x^{*}, q^{*}, p^{*}} \\
& =\left.\left(\sum_{l \in \pi} \tilde{\vartheta}_{l}^{*} \frac{\partial f_{l}}{\partial s_{l}}\right)\right|_{x^{*}, q^{*}, p^{*}}=0
\end{aligned}
$$

and for $P_{l}$, we have

$$
\begin{aligned}
& \left.\left(-\frac{\partial U}{\partial P_{l}}+\sum_{l \in \pi} \tilde{\vartheta}_{l}^{*} \frac{\partial \tilde{g}_{l}}{\partial P_{l}}\right)\right|_{x^{*}, q^{*}, p^{*}}=\left.\left(\sum_{l \in \bar{\pi}} \tilde{\vartheta}_{l}^{*} \frac{1}{f_{l}} \frac{\partial f_{l}}{\partial P_{l}}\right)\right|_{x^{*}, q^{*}, p^{*}} \\
& =\left.\left(\sum_{l \in \pi} \tilde{\vartheta}_{l}^{*} \frac{\partial f_{l}}{\partial P_{l}}\right)\right|_{x^{*}, q^{*}, p^{*}}=0
\end{aligned}
$$

Therefore we have $\left.\left(-\nabla U+\sum_{l \in \pi} \tilde{\vartheta}_{l}^{*} \nabla \widetilde{g}_{l}\right)\right|_{x^{*}, q^{*}, p^{*}}=0$, i.e. $y^{*}, q^{*}$, and $P^{*}$ satisfy the KKT condition for the nonlinear convex problem in (14). Using the same line of analysis we can prove the converse result. The proof is completed.

Note that the optimization problem in (11) is a convex program problem and hence its KKT point is globally optimum. Therefore, we can conclude that the KKT point in P1 is actually globally optimum based on the Lemma 1 and Lemma 2. The following theorem states the main result.

Theorem 2 Let $\mathbf{x}^{*}, \mathbf{q}^{*}$, and $\mathbf{P}^{*}$ denote the limit point of the iterative algorithm with TTSDOA. Then there exists $\alpha^{*}, \gamma^{*} \in \Re^{+}$such that the step size $\alpha<\alpha^{*}$ and $\gamma<\gamma^{*}$, the limit point of $\mathbf{x}^{*}, \mathbf{q}^{*}$, and $\mathbf{P}^{*}$ is the global optimum solution to the problem $P 1$.

\section{CONCLUSIONS}

We have presented a model for the joint design of rate control, scheduling and power control for multihop wireless networks with random access by extending the framework of NUM. We formulate the resource allocation in the network as a utility maximization problem with random scheduling constraints and power control arising from contention for the wireless channel. By dual decomposition, we derive a gradient algorithm with two timescales merit that is not only distributed spatially, but more interestingly, decomposes the system problem vertically into three protocol layers to solve the NUM problem.

\section{REFERENCES}

[1] R. L. Cruz and A. V. Santhanam, "Optimal Routing, Link Scheduling and Power Control in Multihop Wireless Networks," in Proceedings of IEEE INFOCOM, San Francisco, April 2003.

[2] M. Kodialam and T. Nandagopal, "Charaterizing achievable rates in multi-hop wireless networks: the joint routing and scheduling problem," Proc. ACM Mobicom, September 2003.

[3] X. Lin and N. Shroff, "The impact of imperfect scheduling on cross-layer rate control in multihop wireless networks," Proc. IEEE Infocom, 2005.

[4] M. Chiang, "Balancing transport and physical layers in wireless multihop networks: jointly optimal congestion control and power control," IEEE Journal on Selected Areas in Communications, vol.23, no. 1, pp. 104-116, Jan. 2005.

[5] X. Wang and K. Kar, "Cross-layer rate control for end-to-end proportional fairness in wireless networks with random access", MobiHoc'05, Urbana-Champaign, Illinois, USA, May 2005. 